\title{
Metastasiertes Mammakarzinom
}

\section{Teil 2}

ährend das erste Heft eher der „allgemeinen“, systemischen Therapie des metastasierten Mammakarzinoms gewidmet ist, werden Sie in dieser Ausgabe eine Reihe an "speziellen"Behandlungsoptionen finden.

Überleitend vom ersten Heft findet sich ein Beitrag zum Stellenwert der Chemosensitivitätstestung. Es folgen Artikel zu lokoregionären, organbezogenen Behandlungsoptionen wie intraarterielle Chemotherapie zur Behandlung des Lokalrezidivs, laserinduzierte Thermotherapie zur Behandlung von Lebermetastasen und Gamma-knife zur Behandlung von zerebralen Metastasen. Wegen des hohen praktischen Stellenwerts folgt eine ausführliche Darstellung der Therapiemöglichkeiten bei ossärer Metastasierung.

Das Heft wird last but not least abgerundet durch einen Beitrag, der sich mit den begleitenden Therapiemaßnahmen, die oft denselben Stellenwert wie die Kausaltherapie haben werden, beschäftigt.

Insgesamt haben die meisten in diesem Heft beschriebenen

Behandlungsoptionen sicher nicht den Status des Standardverfahrens. Als Reservemethoden jedoch kann ihre Anwendung im Einzelfall segensreich sein. Die Indikationsstellung ist mehr noch als bei den im ersten Heft beschriebenen Therapieoptionen an den individuellen Verlauf geknüpft und stellt hohe Ansprüche an die interdisziplinäre Zusammenarbeit. Die Hoffnung, hier im Sinne unserer Patientinnen eine Beitrag geleistet zu haben, möge den Leser beim Studium diese Heftes begleiten.

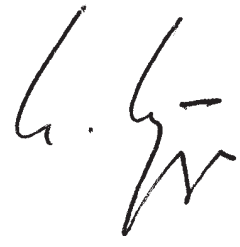

Prof. H. Hepp

Direkor der Frauenklinik

Klinikum Großhadern, München

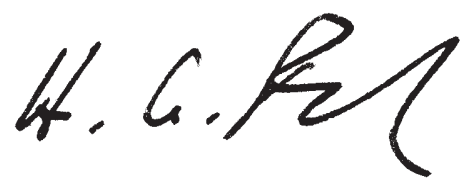

Prof. H.G. Bender

Direkor der Frauenklinik

Med. Universitätsklinik, Düsseldorf

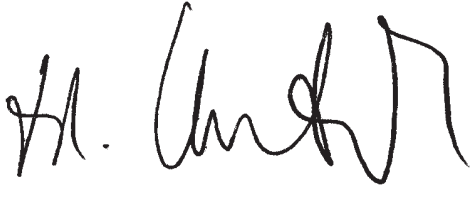

Dr. M. Untch

Oberarzt der Frauenklinik

Klinikum Großhadern, München

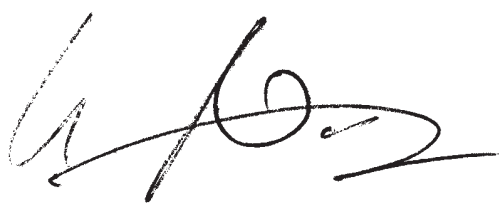

Dr. U. Nitz

Oberärztin der Frauenklinik

Med. Universitätsklinik, Düsseldorf 